\title{
ROS-independent Nrf2 activation in prostate cancer
}

Research Paper

\author{
Ilaria Bellezza ${ }^{1}$, Paolo Scarpelli ${ }^{1}$, Salvatore V. Pizzo ${ }^{2}$, Silvia Grottelli ${ }^{1}$, Egidia \\ Costanzi ${ }^{1}$ and Alba Minelli ${ }^{1}$ \\ ${ }^{1}$ Department of Experimental Medicine, University of Perugia, Perugia, Italy \\ ${ }^{2}$ Duke University School of Medicine, Durham, NC, USA \\ Correspondence to: llaria Bellezza, email: ilaria.bellezza@unipg.it \\ Paolo Scarpelli, email: paolo.scarpelli@unipg.it
}

Keywords: GRP78/BiP, Nrf2, Akt, Anti-GRP78/BiP antibody, dexamethasone

Received: April 12, $2017 \quad$ Accepted:May 23, $2017 \quad$ Published: June 28, 2017

Copyright: Bellezza et al. This is an open-access article distributed under the terms of the Creative Commons Attribution License 3.0 (CC BY 3.0), which permits unrestricted use, distribution, and reproduction in any medium, provided the original author and source are credited.

\section{ABSTRACT}

In prostate cancer, oxidative stress and the subsequent Nrf2 activation promote the survival of cancer cells and acquired chemoresistance. Nrf2 links prostate cancer to endoplasmic reticulum stress, an event that triggers the unfolded protein response, aiming to restore cellular homeostasis as well as an adaptive survival mechanism. Glucose-regulated protein of $78 \mathrm{kD} /$ immunoglobulin heavy chain binding protein (GRP78/BiP) is a key molecular chaperone in the endoplasmic reticulum that, when expressed at the cell surface, acts as a receptor for several signaling pathways enhancing antiapoptotic and proliferative signals. We showed GRP78/ BiP translocation to PC3 cell surface in the presence of tunicamycin, an ER stress inductor, and demonstrated the existence of a GRP78/BiP-dependent non-canonical Nrf2 activation, responsible for increased resistance to ER-stress induced apoptosis. We found that, even in the absence of ROS production, tunicamycin causes Nrf2 activation, and activates Akt signaling, events bulnted by anti-GRP78/BiP antibody treatment. The presence of GRP78/BiP at the cell surface might be exploited for the immunotherapeutic strategy of prostate cancer since its blockage by anti-GRP78/BiP antibodies might promote cancer death by suppressing some of the several molecular protective mechanisms found in aggressive cancer cells.

\section{BACKGROUND}

Oxidants and oxidative stress-inducing agents activate the transcription factor NF-E2-related factor 2 (Nrf2) which controls the fate of the cell by up-regulating the transcription of stress-response genes thus enhancing the antioxidant cell defense and maintaining cellular redox homeostasis [1]. Elevated levels of reactive oxygen species (ROS) in normal cells are harmful and can lead to the induction of cell death by necrosis and/or apoptosis. However, acute and short exposure to high levels of ROS or chronic exposure to low levels of ROS can increase cell proliferation and accelerate tumorigenesis by altering the expression of growth factors and protooncogenes [2-4]. In prostate cancer (PCa), oxidative stress is one of the several hallmarks of the aggressive phenotype since oxidative stress is associated with $\mathrm{PCa}$ development, progression and the response to therapy [5]. Therefore, activation of Nrf2 pathway, leading to an adaptive response, was firstly proposed as a promising strategy for cancer prevention [6]. However, the negative results of several antioxidant-supplemented clinical trials [7], questioned the relationship between oxidative stress and the activation of survival pathways in malignant prostate. Furthermore, Nrf2 protects not only normal cells from transforming into cancer cells, but also promotes the survival of cancer cells [8]. Moreover, Nrf2 and its downstream genes are over-expressed in many cancer cell lines and human cancer tissues, as well as being upregulated in resistant cancer cells and responsible for 
acquired chemoresistance [9-10]. Nrf2 and its activation, besides driving the effects of cellular oxidants and toxic compounds in the cells, as a direct substrate of the PERK branch of the unfolded protein response [11] definitely links PCa to endoplasmic reticulum (ER) stress [12-13].

Prostate cancer is one of the most common cancer in male population, representing $20 \%$ of the newly diagnosed malignancies in Italy in 2015 [14]. Androgen ablation is the standard therapeutic option which leads to an initial regression followed, in the vast majority of the cases, by a tumor relapse into castration resistant $\mathrm{PCa}$ (CRPC), a particularly aggressive phenotype for which there is currently no therapeutic treatment available [15]. PCa, as a solid tumor, deals with events that, by interfering with the ER, lead to ER stress [15]. To restore ER homeostasis, the cells activate several signaling pathways, known as the unfolded protein response (UPR). UPR activation represents an adaptive survival mechanism [16]. In PCa, UPR marker gene activation and tumor progression are linked either by a negative correlation in model systems in vitro [12], or by a positive association that involves androgens and androgen receptor (AR) [17]. Among the several players of UPR, glucose-regulated protein of $78 \mathrm{kD}$ /immunoglobulin heavy chain binding protein (GRP78/BiP) is a key molecular chaperone in the ER, where it presides the folding and assembly of newly synthesized proteins. Elevated levels of GRP78/ BiP characterize several cancer cell lines and human cancers with a close association with metastases and resistance to chemotherapy [18]. Indeed, under ER stress conditions, it can be expressed at the cell surface, acting as a receptor for several signaling pathways that control/ enhance antiapoptotic and proliferative signals [19-20]. AR negative PC3 cells, treated with tunicamycin (TM), up-regulated GRP78/BiP mRNA levels [21], and, when exposed to thapsigargin, relocalised GRP78/BiP on the membrane [22].

In the present study, we showed GRP78/BiP translocation to the cell surface in the presence of TM, an ER stress inductor. We aim to investigate whether GRP78/ $\mathrm{BiP}$ translocation is responsible for PC3 resistance to cell death via a non-canonical Nrf2 activation.

\section{RESULTS}

\section{Tunicamycin causes Nrf2 activation in the absence of increased levels of ROS}

Protein folding occurring in the ER drives the production of reactive oxygen species (ROS), which, in turn, can cause ER stress and trigger the UPR, one of the several pathogenetic mechanisms of prostate cancer initiation and progression [23-24]. To investigate the molecular mechanism underlying the aggressive disease phenotype, we used the AR negative PC3 cell line and studied their response to the treatment with increasing concentrations of tunicamycin (TM) (Figure 1). We observed a moderate decrease in cell viability (Figure 1A) as well as a moderate increase in the number of apoptotic cells (Figure 1B), both suggestive of a mild toxicity of the ER stressor. The clonogenic assay confirmed the presence of viable PC3 cells after treatment with $5 \mu \mathrm{g} / \mathrm{ml}$ TM (Figure 1C). Given that oxidative stress is one of the hallmarks of the aggressive phenotype [2-4], and the existence of a cross talk between UPR and Nrf2 [11], we then analyzed the effects of TM treatment on ROS production (Figure 1D), nuclear translocation (Figure 1E) and transcriptional activity of Nrf2 (Figure 1F), and transcription of Nrf2-master genes (Figure 1G). TM did not increase ROS production while causing a robust Nrf2 activation and the up-regulation (approx. 2 folds) of Nrf2-driven genes Hemeoxygenase-1 (HO-1) and NADPH-quinone oxidoreductase-1 (NQO1). High levels of basal nuclear Nrf2 were observed in PC3, as compared with MDAPCa2b, an androgen sensitive cell line (Supplementary Figure 1A). Results support the activation of the redox-sensitive transcription factor Nrf2 as one of several culprits of cancer cell death.

\section{Tunicamycin induces the activation of the IRE1 $\alpha$ arm}

The highly integrated and regulated UPR signal transduction pathways are triggered by three proteins residing in the ER membrane: inositol requiring-enzyme 1 alpha (IRE1 $\alpha$ ), activating transcription factor 6 alpha (ATF6 $\alpha$ ) and protein kinase RNA-like ER kinase (PERK). These ER sensors, under normal and physiological conditions, are kept in an inactive state by GRP78/BiP, which, upon several ER stressing stimuli, dissociates from the sensors and activates the UPR signaling pathways [16]. PC3 cells treated with TM showed a late increase in GRP78/BiP levels, probably indicative of the activation of the ATF6 $\alpha$ branch, absence of PERK-mediated phosphorylation of eif $2 \alpha$ at each considered time, whereas phosphorylation of IRE1 $\alpha$ was detected at 2-3h and remained sustained up to $24 \mathrm{~h}$ (Figure 2A). Results indicate the activation of the adaptive IRE $1 \alpha$ and ATF $6 \alpha$ branches of the UPR while simultaneously inhibiting the PERK pathway.

\section{Tunicamycin-induced IRE1 $\alpha$ activation fails to activate MAPK cascades}

Phosphorylated IRE1 $\alpha$ acts as a stress-specific scaffold on the cytosolic side of ER and associates with TRF-receptor associated factor 2 (TRAF2) to activate several kinases, which, in turn, contribute to cell fate decision during ER stress by acting on Nrf2 and NF$\kappa \mathrm{B}$ [23]. The treatment of PC3 cells with TM failed to activate extracellular signal-regulated kinase (ERK), 
A

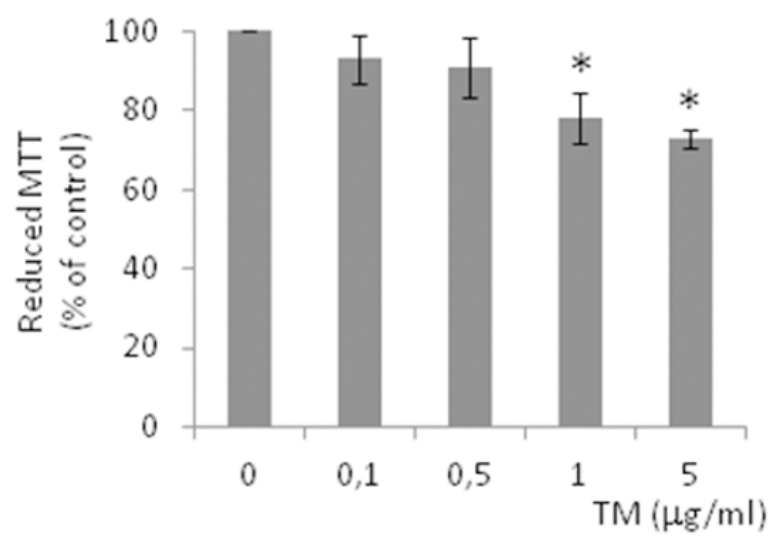

C

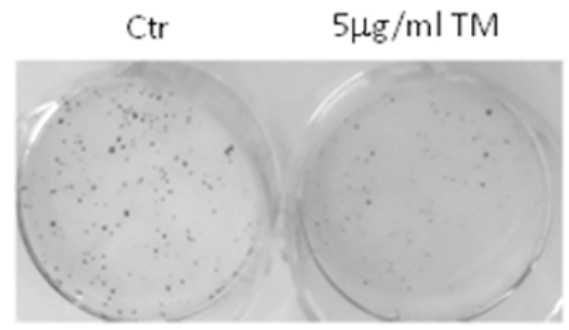

$E$ $\begin{array}{lllllll}0 & 1 & 2 & 3 & 6 & 24 & \text { Time }(\mathrm{h})\end{array}$

\begin{tabular}{lllllll}
$\mathrm{Nrf} 2 / \mathrm{H} 3$ & 0.37 & 0.45 & 0.28 & 1.11 & 0.98 & 0.87 \\
\hline
\end{tabular}

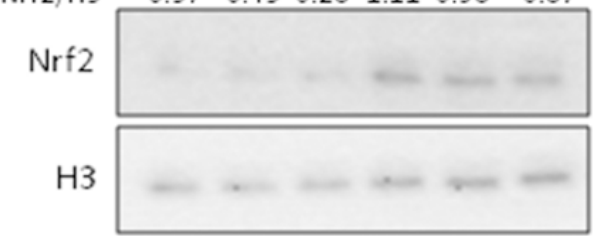

G

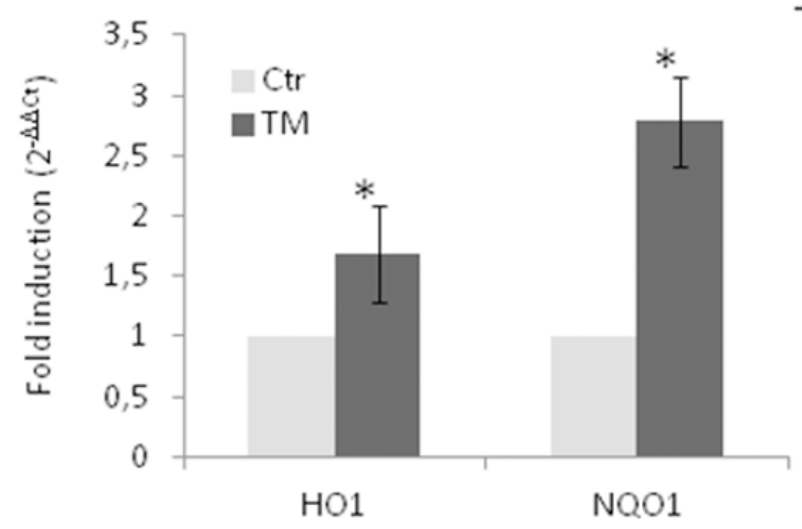

B
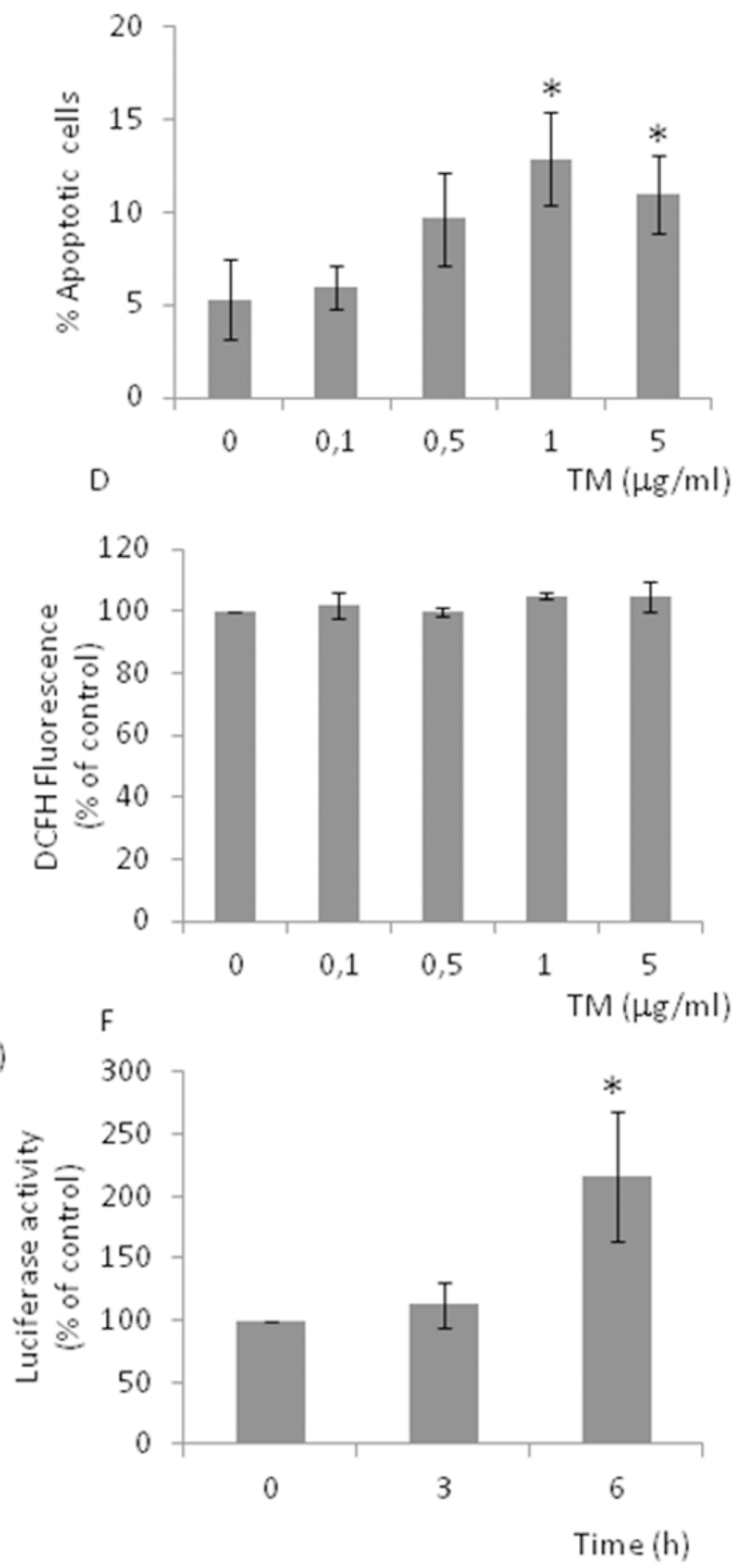

Figure 1: Tunicamycin causes Nrf2 activation in the absence of increased levels of ROS. PC3 cells were treated with increasing concentrations $(0-5 \mu \mathrm{g} / \mathrm{ml})$ of TM for $24 \mathrm{~h}$. (A) Cell viability as detected by MTT assay; (B) percentage of apoptotic cells as detected by PI staining and FACS analysis; (C) clonogenic assay, in the presence of $5 \mu \mathrm{g} / \mathrm{ml}$ TM; (D) ROS levels as detected by DCFH fluorescence; (E) Nrf2 nuclear levels as detected by western blotting, histone H3 was used as loading control. (F) Nrf2 activation as detected by luciferase assay. Control values (mean \pm S.D., $\mathrm{n}=6$ ) are given as $100 \%$. (G) HO-1 and NQO-1 expression as determined by qPCR. Expression was normalised to GAPDH and reported as $2^{-\Delta \Delta \mathrm{Ct}}$. Relative mRNA level of untreated cells was assumed to be 1 . $* \mathrm{p}<0.05$ vs. control cells. 
c-Jun N-terminal kinase (JNK), p38 MAPK, and NF$\kappa \mathrm{B}$ (Figure 2B), indicating that the activation of all three MAPK cascades were not responsible for Nrf2 modulation.

\section{Tunicamycin activates Akt signaling}

The phosphatidylinositol 3'-kinase (PI3K)/Akt signaling pathway regulates cell survival during oxidative stress by activating Nrf2 [25-26]. Given that Akt is overexpressed in prostate cancers [27] and that activated Akt promotes cell survival [28-29], to better understand the mechanism underpinning Nrf2 activation, we investigated the effects of TM treatment on Akt signaling pathway (Figure 2C). We found that TM increased the levels of phosphorylated Akt with a time-course indicating a maximal activation at $3 \mathrm{~h}$ lasting up to $6 \mathrm{~h}$. Concomitantly, we observed GSK $3 \beta$ phosphorylation, thus inhibiting the apoptosis-inducing kinase activity. Pretreatment of the cells with wortmannin, a PI3-kinase inhibitor, showed that activation of Akt and inactivation of GSK $3 \beta$ are PI3K dependent. These results indicate that Nrf2 activation, under our experimental condition, can, at least partly, be attributed to the activation of Akt.
A

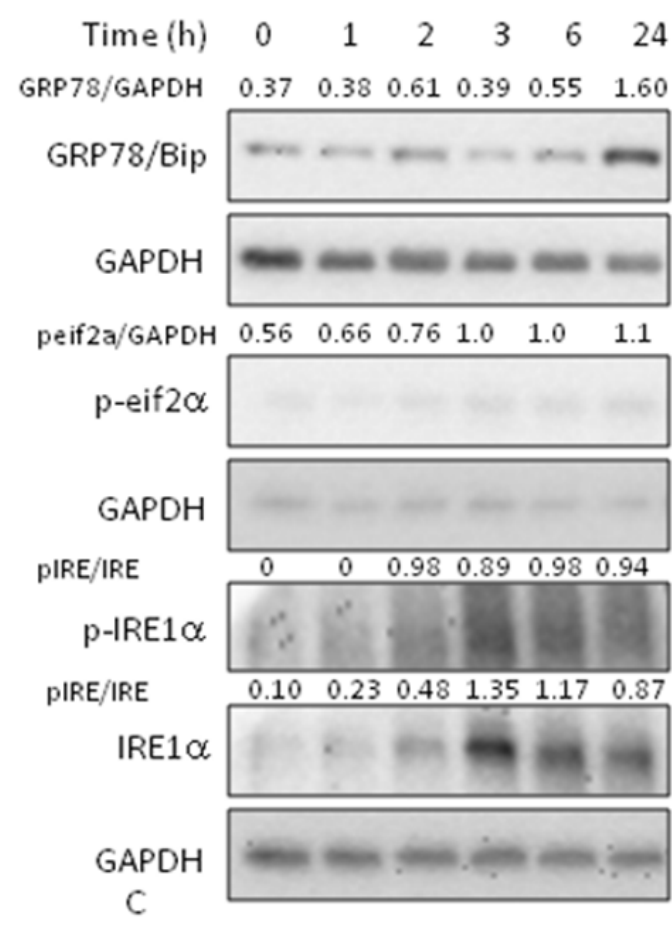

B

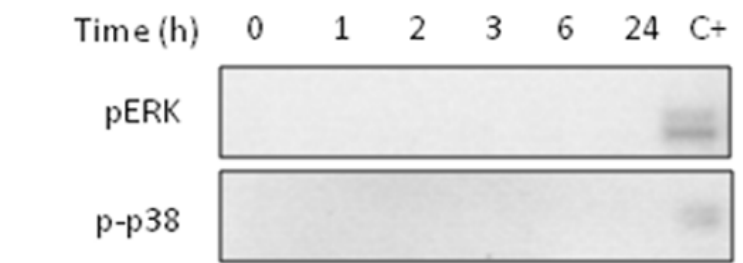

GAPDH

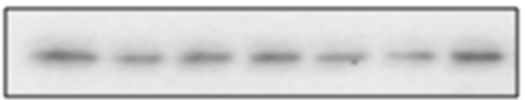

p-SAPK/JNK

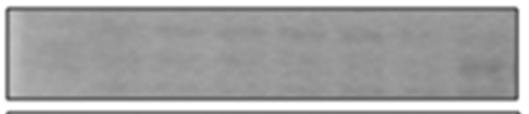

GAPDH

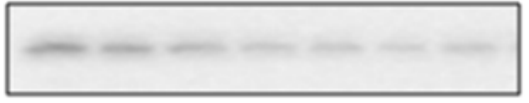

pNF-KB/GAPDH $\quad 1.66 \quad 1.93 \quad 2.00 \quad 1.51 \quad 1.26 \quad 0.82$

pNF-KB

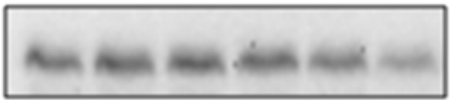

GAPDH

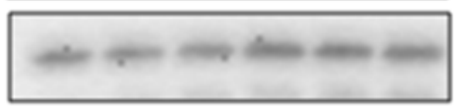

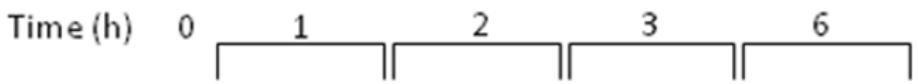

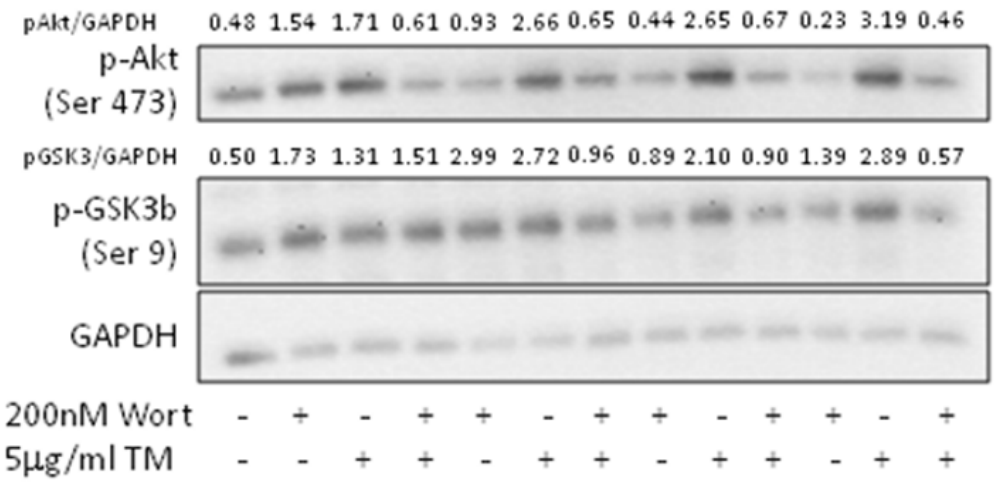

Figure 2: Tunicamycin activates AKT signaling. PC3 cells were treated with $5 \mu \mathrm{g} / \mathrm{ml} \mathrm{TM}$ for the indicated time, collected and total extract subjected to western blotting with the indicated antibodies. Wortmannin was added to cell culture $1 \mathrm{~h}$ prior to TM treatment. (A) Analysis of UPR markers; (B) analysis of MAPK activation; (C) analysis of Akt pathway activation. GAPDH was used as loading control. One out of four independent experiments giving similar results is shown. 


\section{Tunicamycin treatment induces the translocation of GRP78/Bip to the cell surface}

GRP78/BiP is a resident ER chaperone which appears at the surface of many cancer cells, where it is involved in the activation of pro-proliferative/anti-apoptotic signaling mechanisms [30-33]. Although it is known that PC 3 cells do not express GRP78/BiP on their cell surface, thapsigargin treatment was shown to induce GRP78/BiP membrane localization [22]. Therefore, we anticipated that the treatment of PC3 with TM could also relocalizeGRP78/ $\mathrm{BiP}$ on the cell surface. We found that a $3 \mathrm{~h}$ treatment with TM caused GRP78/BiP translocation to the cell membrane, thus suggesting a mechanism for $\mathrm{PC} 3$ refractoriness to TM (Figure 3A). To support the hypothesis that translocation of GRP78/BiP to the cell surface is responsible for increased survival of the cells and drug resistance, we used AR positive MDAPCa2b cells that, when treated with TM, responded with decreased cell survival, increased apoptosis, and increased ROS production (Supplementary Figure 1B1E). Indeed, in MDAPCa2b treated with TM, we did not observe GRP78/BiP translocation to the membrane (Figure 3B). The treatment of PC3 cells with $1 \mu \mathrm{g} / \mathrm{ml} \mathrm{TM}$ caused GRP78/BiP translocation at the cell surface (Figure 4A) and Nrf2 activation (Figure 4B), thus confirming that the observed effects were not due to tunicamycin toxicity. The secretory pathway, representing the first step in the protein export from the ER, is mediated by coatomer protein II (COPII)-coated vesicles at ER exit sites. The conserved large hydrophilic protein Sec16 localizes at the ER exit sites and plays a pivotal role in modulating the COPII dynamics [34]. In PC3 and MDAPCa2b cells treated with TM, we observed the up-regulation of Sec16L mRNA levels, thus confirming the activation of the secretory pathway by TM (Supplementary Figure 1F).

\section{Anti-GRP78/BiP C-terminal domain antibody treatment blunts Nrf2 activation}

The activation of antioxidant and anti-apoptotic signaling is a protective response since it establishes a shield over death signals in cancer cells. The translocation of GRP78/Bip to the cell surface of PCa leads to cell survival and proliferation, thus contributing to masking several stresses [28-29, 35]. To determine whether Nrf2 activation and the subsequent redox adaptation, a strategy frequently used by cancer cells to survive and become resistant to several anticancer agents, might be related to the response of $\mathrm{PC} 3$ cells to TM treatment, we exposed PC3 cells to GRP78/BiP-antibody and investigated the effects of TM. Under these experimental conditions, we found an increase in ROS levels (Figure 5A), changes in the phosphorylation of Akt and GSK3 $\beta$, indicative of a reduced activation of Akt (Figure 5B). Moreover, the Nrf2

A
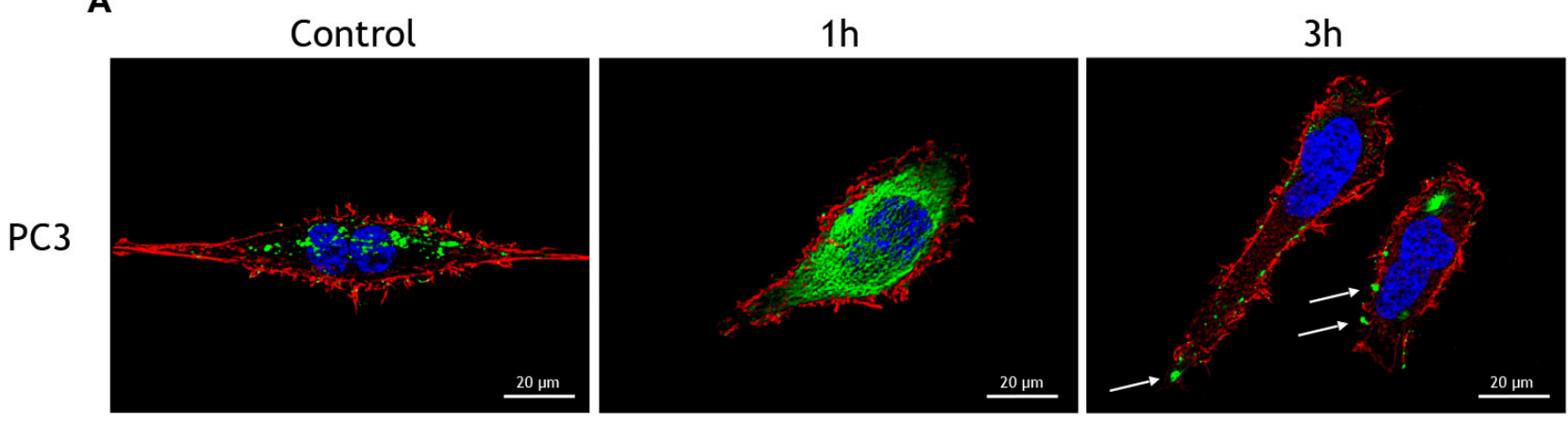

B

Control

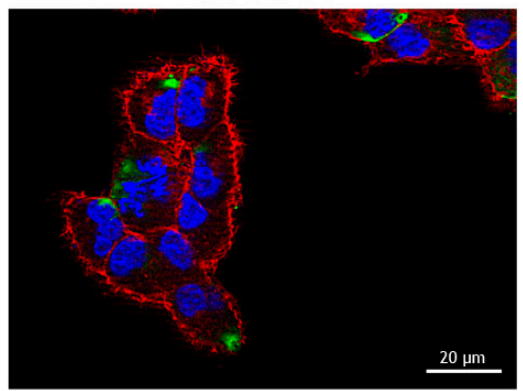

$1 \mathrm{~h}$

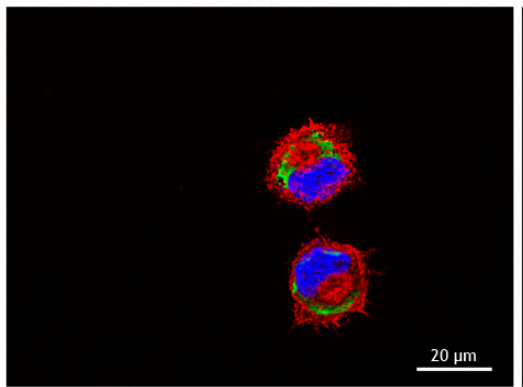

$3 h$

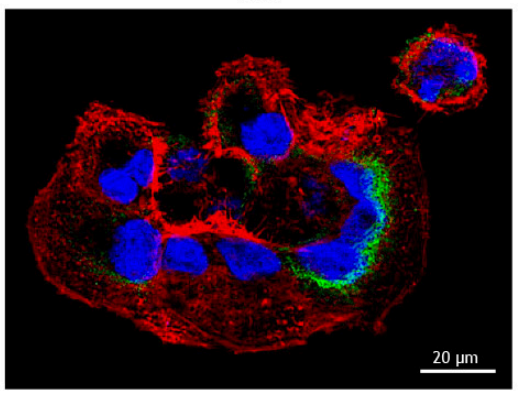

Figure 3: Tunicamycin treatment induces the translocation of Bip to the cell surface. (A) PC3 cells and (B) MDAPCa2b cells were treated with $5 \mu \mathrm{g} / \mathrm{ml} \mathrm{TM}$ for the indicated times and used for detection of GRP78/BiP (green) by immunofluorescence. Cytoskeletal actin was visualized by AlexaFluor555-conjugated phalloidin staining (red) and nuclei were counterstained with DAPI (blue). Magnification: $63 \times$. 
transcriptional activity reverted to control values (Figure 5C) as well as Nrf2-driven gene expression (Figure 5D). Results indicate that Nrf2 activation can be, at least partially, due to GRP78/BiP translocation.

\section{Dexamethasone treatment of PC3 cells do not affect GRP78/BiP translocation}

The combination of chemotherapy and dexamethasone is a classic treatment for CRPC patients [36] thus we investigated the effects of dexamethasone treatment on TM-treated PC3 cells. We found that PC3 cells still exposed GRP78/BiP on their surface (Figure 5A) and that Akt pathway was still active (Figure 5B) as well as Nrf2 (Figure 6C) resulting in unchanged ROS levels (Figure 6D). The results indicate that dexamethasone treatment does not modify Nrf2 adaptive responses.

\section{DISCUSSION}

Here we have shown in PC3 cells, an AR negative $\mathrm{PCa}$ cell line, that translocation of GRP78/BiP to the cell surface enhances Nrf2 activation thus contributing to cells survival. Cancer is a complex biological process characterized by several hallmarks, i.e. biological capabilities that increase survival by evading cell death signaling and immune destruction in a new tissue microenvironment [37]. PCa is the second-leading cause of cancer-related mortality in men in Western countries, representing $20 \%$ of the newly diagnosed malignancies in Italy in 2015 [14]. PCa initially responds to surgical or chemical depletion of the AR ligand. However, castration induces several adaptive responses which, combined with genomic and epigenomic mutations [3839], lead to the progression, in approx. 18 months, to
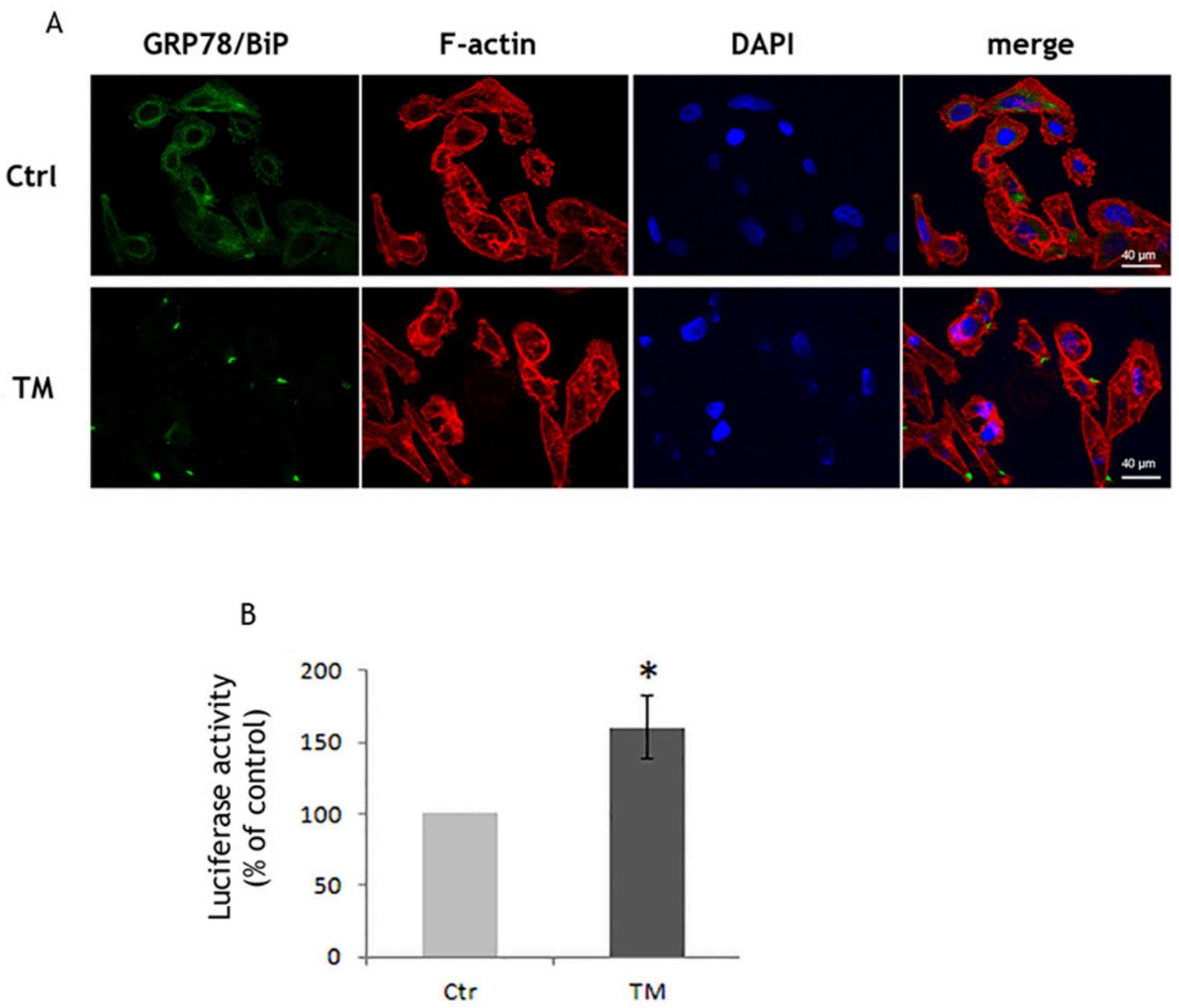

Figure 4: Low tunicamycin concentration induces the translocation of Bip to the cell surface. PC3 cells were treated with $1 \mu \mathrm{g} / \mathrm{ml} \mathrm{TM}$ and (A) after $3 \mathrm{~h}$ used for the detection of GRP78/BiP (green) by immunofluorescence. Cytoskeletal actin was visualized by AlexaFluor555-conjugated phalloidin staining (red) and nuclei were counterstained with DAPI (blue). Magnification: 40×; (B) after 6h used for the determination of Nrf2 activation by luciferase assay. Control values (mean \pm S.D., $\mathrm{n}=6$ ) are given as $100 \%$. 


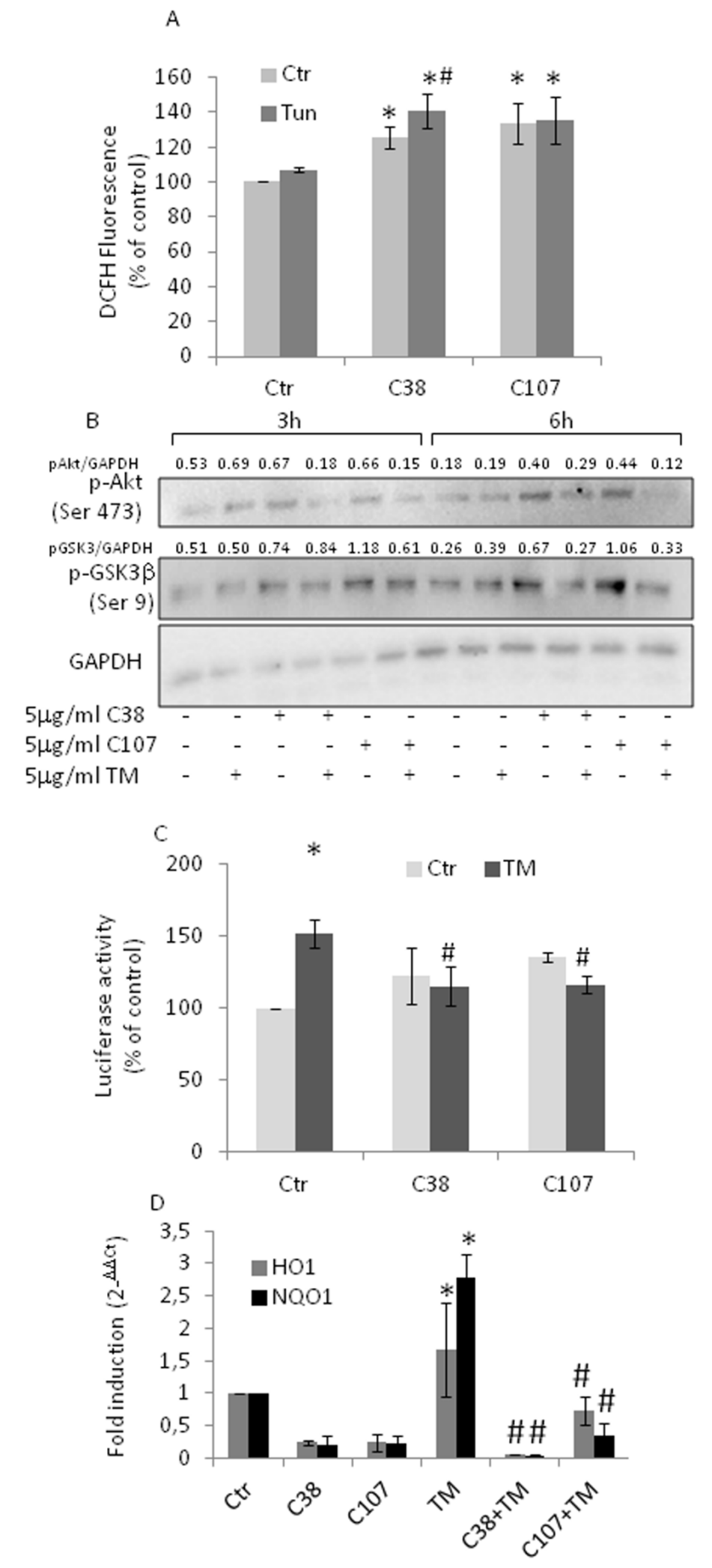

Figure 5: Anti- GRP78/BiP C-terminal domain antibody treatment blunts Nrf2 activation. PC3 cells were treated with $5 \mu$ g/ $\mathrm{ml}$ of TM $10 \mathrm{~min}$ prior to antibody addition $(5 \mu \mathrm{g} / \mathrm{ml} \mathrm{C38}$ or C107) and grown for the indicated times. (A) ROS levels as detected by DCFH fluorescence. (B) Total cell extract subjected to western blotting with the indicated antibodies. GAPDH was used as loading control. One out of four independent experiments giving similar results is shown. (C) Nrf2 activation as detected by luciferase assay; Control values (mean \pm S.D., $\mathrm{n}=4$ ) are given as 100\%. (D) HO-1 and NQO-1 expression as determined by qPCR. Expression was normalised to GAPDH and reported as $2^{-\Delta \Delta C t}$. Relative mRNA level of untreated cells was assumed to be 1 . ${ }^{*} \mathrm{p}<0.05$ vs. control cells; \# $<<0.05$ vs. TM-treated cells. 

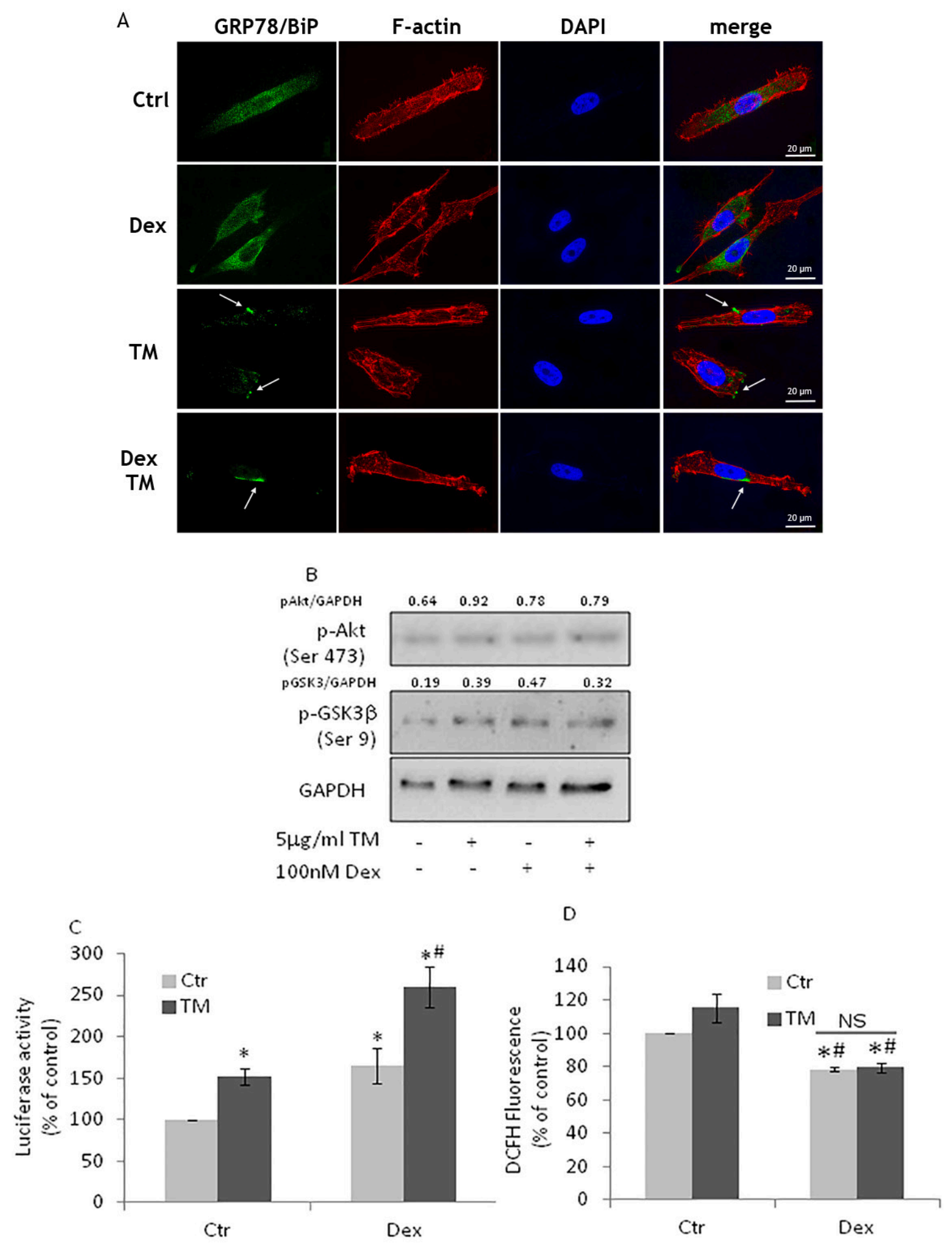

Figure 6: Dexamethasone treatment of PC3 cells does not affect GRP78/BiP translocation. (A) PC3 cells, treated with100nM Dexamethasone (Dex) for $72 \mathrm{~h}$, were exposed to $5 \mu \mathrm{g} / \mathrm{ml} \mathrm{TM}$ for $3 \mathrm{~h}$ and used for detection of GRP78/BiP (green) by immunofluorescence. Cytoskeletal actin was visualized by AlexaFluor555-conjugated phalloidin staining (red) and nuclei were counterstained with DAPI (blue). Magnification: 63×. PC3 cells, after Dex treatment, were exposed to TM for $6 \mathrm{~h}$ and used for (B) western blotting analyses with the indicated antibodies. GAPDH was used as loading control. One out of four independent experiments giving similar results is shown; (C) Nrf2 activation as detected by luciferase assay; (D) PC3 cells, after Dex treatment, were exposed to TM for 24h and ROS levels were detected by DCFH fluorescence; control values (mean \pm S.D., $n=4$ ) are given as $100 \%$. ${ }^{*} \mathrm{p}<0.05$ vs. control cells; $\# \mathrm{p}<0.05$ vs. TM-treated cells. 
a more aggressive phenotype, i.e. CRPC. These cancer cells are provided with a high-grade plasticity, thus a single adaptive pathway is not the only responsible for the apoptosis inhibition and treatment resistance [40]. Among the various adaptive/survival responses, the transcription factor Nrf2 and its network plays a crucial role, going from antioxidant genes to metabolic and proteostasis genes, and autoregulatory pathways [41]. Because of this extended coverage, Nrf2 employs more than one regulatory strategy for transcriptional activation in response to stress. Nrf2 activation is tightly bound to increase on ROS level. Indeed, in normal cell homeostasis, Nrf2 is constantly ubiquitinated by the E3 ligase Keap-1 and degraded. Upon oxidative or electrophilic stress, Nrf2, detached from Keap-1, is translocated to the nucleus, where, by binding to the antioxidant/electrophile response element (ARE/EpRE) in target gene promoters, up-regulates the expression of downstream Nrf2 target genes that coordinate the adaptive responses $[1,42]$. However, Nrf2 activation can be harmful in cancer situations [3, 8, 43]. Mutations that disrupt the Nrf2-Keap-1 interaction, cause a constitutive activation of this transcription factor, and such mutations are observed in lung, colorectal, and prostate cancers [44-46]. Undeniably, the sustained activation of $\mathrm{Nrf} 2$ produces a supportive environment for survival and therapeutic resistance. AR negative PC3 cells, used in this study, are characterized by high basal nuclear levels of Nrf2, a condition that could explain the lack of ROS production as well as the low apoptotic response that we observed after treating PC3 cells with an ER stressor, TM. Nevertheless, under ER stress conditions, we observed an increased transcriptional activity of $\mathrm{Nrf2}$, with a concomitant increase in HO1 and NQO1 gene expression, which could not be attributed to the PERK branch of the UPR pathway. Therefore, we suggest the existence of a non canonical signaling mechanism that may increase Nrf2 activation in the absence of increased levels of ROS under an ER stress setting. As a solid tumor, $\mathrm{PCa}$ often deals with microenvironmental factors that, by compromising the ER function, lead to ER stress. In turn, the stress elicits UPR and all the related cytoprotective mechanisms aimed to favoring tumor growth [23-24]. UPR activation as an adaptive survival mechanism is found in gastric cancer, in malignant glioma, and in chronic lymphocytic leukemia [47-49]. Although at times the relationship between UPR markers and adaptive survival has been debated [12], several studies have shown a positive link between UPR markers and androgen-driven PCa [13, 17, 24]. Moreover, androgens activate the IRE1 $\alpha$-XBP1 arm of UPR and concurrently inhibit the PERK-eIF2 $\alpha$ pathway [17]. In AR negative PC3 cells, we found that TM did not activate the PERK-eIF2 $\alpha$ signaling whereas it increased the protein level of GRP78/BiP, a target gene of the ATF $6 \alpha$ arm, and activated the IRE1 $\alpha$ arm.
Although the role of the IRE1 $\alpha$ pathway in AR-negative cell lines still needs to be further investigated, it is now accepted that the IRE1 $\alpha$ arm plays a pro-survival role in PCa $[17,24]$. The expression of molecular chaperones provides numerous types of cancer with pro-survival aptitude [50]. In PCa, GRP78/BiP is one of the most studied molecular chaperone and the increases in its expression are correlated with $\mathrm{PCa}$ recurrence and short survival [51-52]. In several cancers, as well as in PCa, GRP78/BiP translocates to cell surface thus constituting a cancer-specific cell surface marker, tightly related to hormonal resistance of $\mathrm{PCa}[28,33]$. The PI3K/Akt pathway is significantly involved in multiple biological responses that promote survival thus the increased phosphorylation of Akt in prostate cancer tissues frequently prevents apoptosis and contributes to tumor progression [53]. GRP78/BiP, when expressed at the cell surface of tumor cells, enhances the Akt signaling thus contributing to cell survival and aggressive phenotype. Indeed, the highly metastatic $1-\mathrm{LN}$ cells, derived from less metastatic PC3 cells, express GRP78/BiP on their cell surface $[22,29,35]$. By treating PC3 cell with TM, we therefore obtained cells with a distinctive marker of high metastatic potential. Given previous studies, activation of surface-bound GRP78/BiP will trigger the activation of Akt, which by inactivating GSK3 $\beta$, leads to the reinforcement of Nrf2-mediated defense mechanism. Our study, by using wortmannin, a PI3K inhibitor, confirms that the role of Akt in PC3 is linked to the activation of the Nrf2/ARE transcriptional regulation in the presence of translocated GRP78/BiP. Indeed, when we specifically targeted cell surface GRP78/BiP with C-terminal directed antibodies, we observed a decreased Nrf2 activation, concomitant to a decreased activation of Akt and a decreased inactivation of GSK3 $\beta$. The active GSK3 $\beta$ enhances Nrf2 degradation thus weakening PC3 cellular defense. Our proposal, i.e. that translocated GRP78/BiP could enhance Nrf2 activation, was substantiated by using MDAPCa2b cell line, characterized by the lack of GRP78/BiP translocation upon TM treatment. These cells responded to the TM treatment with a decreased cell survival, an increased apoptosis, and an increased ROS production due to the lack of Nrf2 activation. Finally, we observed that dexamethasone treatment, commonly used to treat CRPC patients [36], did not abrogate the translocation of GRP78/BiP to the cell surface and every other described cellular response to TM.

In conclusion, the preferential expression of GRP78/ $\mathrm{BiP}$ on the surface of cancer cells might be exploited for immunotherapeutic approaches of prostate cancer. It is known that antibodies to the carboxyl terminal domain of GRP78 promote cancer cell death [22] as also observed in the present study. Indeed, by decreasing Nrf2 activation, this strategy could remove some of the several molecular protective mechanisms found in aggressive cancer cells. 


\section{MATERIALS AND METHODS}

\section{Materials}

All the reagents, unless otherwise stated, were from Sigma-Aldrich (St. Louis, MO). All the antibodies, unless otherwise stated were from Santa-Cruz Biotechnology (Santa Cruz, CA). Antibodies C38 and C107 were validated as described [20].

\section{Cell culture and treatment}

AR negative $\mathrm{PC} 3$ and AR positive MDAPCa2b cell lines were obtained from American Type Tissue Culture Collection (Rockville, MD). Cells were grown in RPMI 1640 medium (Lonza, Milano, Italy) supplemented with $10 \%$ foetal bovine serum (FBS), glutamine $(2 \mathrm{mM})$ and antibiotics (penicillin $100 \mathrm{U} / \mathrm{ml}$ and streptomycin 100 $\mu \mathrm{g} / \mathrm{ml})$. Sub-confluent cells were exposed to increasing concentrations of tunicamycin $(0.1-5 \mu \mathrm{g} / \mathrm{ml})$ for the indicated time. Wortmannin was added to cell culture $1 \mathrm{~h}$ prior to tunicamicyn, anti GRP78/Bip antibodies (C38 and C107) were added 10min after tunicamycin treatment. In some experiments, cells were grown for $72 \mathrm{~h}$ in the presence of dexamethasone and then treated with tunicamycin for the indicated times.

\section{MTT viability assay}

Cell viability was measured using the MTT assay. $0.5 \mathrm{mg} / \mathrm{ml}$ MTT (3-(4,5-dimethylthiazol-2-yl)-2,5diphenyltetrazolium bromide) was added to each well for $4 \mathrm{~h}$, lysis buffer $(10 \% \mathrm{SDS}, 0,01 \mathrm{M} \mathrm{HCl})$ was then added and cells lysed at $37^{\circ} \mathrm{C}$ overnight. Samples were measured with an automatic microplate reader (Seac, Firenze, Italy) at $550 \mathrm{~nm}$. Experiments were performed at least three times with six samples in each experimental group. Results were expressed as percentages (\%) of reduced MTT, assuming the absorbance of control cells as $100 \%$ [54].

\section{Apoptosis determination by flow cytometry}

Apoptosis was detected by propidium iodide (PI) $(50 \mu \mathrm{g} / \mathrm{ml}$ in $0.1 \%$ sodium citrate plus $0.1 \%$ triton $\mathrm{X}-100)$ addition. The PI fluorescence of individual nuclei was measured by flow cytometry using standard FACScan equipment (Becton Dickinson, Franklin Lakes, NJ). The data were recorded in a Hewlett Packard (H9 9000, model 310, Palo Alto, CA) computer. The percentage of apoptotic cell nuclei (sub-diploid DNA peak in the DNA fluorescence histogram) was calculated with specific FACScan research software (Lysis II). At least 10,000 events were analysed in each sample [55].

\section{Measurement of intracellular fluorescence}

The DCFH-DA method was used to detect the levels of intra-cellular reactive oxygen species (ROS). Cells were treated as described and DCFH-DA $(30 \mu \mathrm{M})$ was added into the medium for a further $30 \mathrm{~min}$ at $37^{\circ} \mathrm{C}$. The fluorescence of 2',7'-dichlorofluorescein was detected at $485 \mathrm{~nm}$ excitation and at $535 \mathrm{~nm}$ emission, using a TitertekFluoroscan II (Flow Laboratories, McLean, USA). Results were expressed as \% of the control DCF fluorescence [54].

\section{Western blotting}

Cells were lysed in boiling Laemmli sample buffer or processed with NE-PER(R) Nuclear and Cytoplasmic Extraction Reagents (Pierce Biotechnology, Rockford, IL) according to manufacturer's instruction. Protein samples were electrophoresed on SDS polyacrylamide gels and transferred to nitrocellulose membranes at 100 $\mathrm{V}$ for $1 \mathrm{hr}$. Membranes were probed with the indicated antibodies (Supplementary Table 1), which were detected using HRP-based chemiluminescence (ECL, Pierce Biotechnology, Rockford, IL) [56]. Densitometric analises were performed with Image J software.

\section{Clonogenic assay}

Cells were seeded at a density of 500 cells/dishes in $9.5 \mathrm{~cm}^{2}$ well. After a 7 days incubation at $37^{\circ} \mathrm{C}$, the colonies were fixed with $3.7 \%$ paraformaldehyde, stained with crystal violet $(0.5 \% \mathrm{w} / \mathrm{v})$ and counted using a stereomicroscope.

\section{Immunofluorescence}

Cells, seeded on glass coverslips, were fixed with $4 \%$ paraformaldehyde in PBS (Chem Cruz) for $20 \mathrm{~min}$ at room temperature. After blocking non-specific binding sites with 3\% BSA in PBS for $1 \mathrm{~h}$, cells were incubated overnight at $4{ }^{\circ} \mathrm{C}$ with anti GRP78 antibody (C38) $(1: 100)$ and then with anti mouse Alexafluor 488 secondary antibody (1:500). After washings, F-actin was stained with Alexafluor 555-Labelled phalloidin (1:200) for 30 minutes at room temperature. Cells were washed with PBS, cell nuclei were counter-stained with 4,6-diamidino2-phenylindole (DAPI) and slides were mounted using glass coverslips and Prolong Diamond mounting medium (Life Technologies) for permanent sealing. Images were captured using a Zeiss Axio Observer. Z1 inverted microscope, equipped with Apotome filter and Axiocam MRm camera detection system Zeiss using a $63 \mathrm{x} / 1.25$ oil Plan/neofluar objective and a 40x/0.75 plan/neofluar objective. To improve axial resolution and focal planes separation, Apotome filter was enabled, set to "strong" with noise reduction set to "Off". All the images, were 
processed for visual improvement, with the application of a 5,6 pixel 160 point sharpening mask and histograms were additionally stretched to increase contrast (Ps CC Adobe). No in-silico extra noise reduction treatment was applied to the images.

\section{RNA isolation, reverse transcription and RT- PCR}

Total RNA was isolated with TRI Reagent according to the manufacturer's instructions and cDNA was synthesised using PrimeScript RT reagent Kit (Takara, Mountain View, CA). Real time PCR was performed using the QuantStudio 3 detection system (Applied Biosystem, Foster City, CA) and SYBR Green chemistry. Primers are listed in Supplementary Table 2. SYBR Green RT-PCR amplifications were carried out in a 96-well plate in a $25 \mu \mathrm{l}$ reaction volume that contained $12.5 \mathrm{ml}$ of $\mathrm{SYBR}^{\circledR}$ Green JumpStart ${ }^{\mathrm{TM}}$ Taq ReadyMix, $400 \mathrm{nM}$ forward and reverse primers, and 5 to $40 \mathrm{ng}$ of cDNA. In each assay, no-template controls were included and each sample was run in triplicates. Mean of $\mathrm{Ct}$ values of the samples was compared to the untreated control sample and GAPDH used as internal control. The n-fold differential ratio was expressed as $2^{-\Delta \Delta \mathrm{Ct}}$.

\section{Transfection and luciferase reporter assays}

Cignal ARE Dual-luciferase reporter kit (CCS5020, Qiagen, Milan, IT) was used to transfect PC3 and MDA cells following manufacturer's instructions. Transient transfections were carried out using Attractene transfection reagent (Qiagen, Milan, IT) according to the manufacturer's instructions. Cell extracts were prepared $24 \mathrm{~h}$ after transfection and Luciferase activities in lysates were measured using Luminoskan (Thermo Electron Corporation, Waltham, MA) luminometer. Quantification of Firefly and Renilla luciferase activities was performed with the Dual Luciferase Reporter Assay System (Promega, Madison, WI). The relative firefly luciferase activity was calculated by normalizing transfection efficiency. The luciferase activity was expressed as arbitrary unit of light intensity.

\section{Statistical analysis}

All studies were performed at least in triplicate, separate experiments and expressed as mean \pm S.D. Data were analysed for statistical significance by Student's t-test. When appropriate data were analysed by twoway ANOVA test with Sidak's correction for multiple comparison. ${ }^{*} \mathrm{P}$-values $<0.05$ were considered significant.

\section{Abbreviations}

AR: androgen receptor; ARE/EpRE: antioxidant/ electrophile response element; ATF6 $\alpha$ : activating transcription factor 6 alpha; COPII: coatomer protein II; CRPC: castration resistant PCa; ER : endoplasmic reticulum; ERK: extracellular signal-regulated kinase; GRP78/BiP: glucose-regulated protein of $78 \mathrm{kD} /$ immunoglobulin heavy chain binding protein; HO-1: Hemeoxygenase-1; IRE1 $\alpha$ : inositol requiring-enzyme 1 alpha; JNK: c-Jun N-terminal kinase; NQO1: NADPHquinone oxidoreductase-1; Nrf2: NF-E2-related factor 2; PCa: prostate cancer; PERK: protein kinase RNA-like ER kinase; PI3K: phosphatidylinositol 3'-kinase; ROS: reactive oxygen species; TM: tunicamycin; TRAF2: TRF-receptor associated factor 2; UPR: unfolded protein response.

\section{Authors' contributions}

IB: acquired and interpreted data; PS: performed the immunofluorescence examinations, SG, EC: performed some experiments; AM and IB: conceived and wrote the MS; SVP: critically revised the MS. All authors read and approved the final manuscript.

\section{CONFLICTS OF INTEREST}

The Authors declare no competing interest.

\section{FUNDING}

This study was supported by Fondazione Cassa di Risparmio di Perugia (2015.0326.021). The funders had no role in the study design, data collection and analysis, decision to publish, or preparation of the manuscript.

\section{REFERENCES}

1. Brigelius-Flohé R, Flohé L. Basic principles and emerging concepts in the redox control of transcription factors. Antioxid Redox Signal. 2011; 15:2335-81.

2. Klaunig JE, Kamendulis LM, Hocevar BA. Oxidative stress and oxidative damage in carcinogenesis. Toxicol Pathol. 2010; 38:96-109.

3. Minelli A, Bellezza I, Conte C, Culig Z. Oxidative stressrelated aging: A role for prostate cancer? Biochim Biophys Acta. 2009; 1795:83-91.

4. Paschos A, Pandya R, Duivenvoorden WC, Pinthus JH. Oxidative stress in prostate cancer: changing research concepts towards a novel paradigm for prevention and therapeutics. Prostate Cancer Prostatic Dis. 2013; 16:217-25.

5. Kumar B, Koul S, Khandrika L, Meacham RB, Koul HK. Oxidative stress is inherent in prostate cancer cells and is required for aggressive phenotype. Cancer Res. 2008; 68:1777-85.

6. Krajka-Kuźniak V, Paluszczak J, Baer-Dubowska W. The Nrf2-ARE signaling pathway: An update on its regulation 
and possible role in cancer prevention and treatment. Pharmacol Rep. 2016; 69:393-402.

7. Jiang L, Yang KH, Tian JH, Guan QL, Yao N, Cao N, Mi $\mathrm{DH}, \mathrm{Wu}$ J, Ma B, Yang SH. Efficacy of antioxidant vitamins and selenium supplement in prostate cancer prevention: a meta-analysis of randomized controlled trials. Nutr Cancer. 2010; 62:719-27.

8. Bellezza I, Grottelli S, Gatticchi L, Mierla AL, Minelli A. $\alpha$-Tocopheryl succinate pre-treatment attenuates quinone toxicity in prostate cancer PC3 cells. Gene. 2014; 539:1-7.

9. Harder B, Jiang T, Wu T, Tao S, Rojo de la Vega M, Tian W, Chapman E, Zhang DD. Molecular mechanisms of Nrf2 regulation and how these influence chemical modulation for disease intervention. Biochem Soc Trans. 2015; 43:680-6.

10. Lau A, Villeneuve NF, Sun Z, Wong PK, Zhang DD. Dual roles of Nrf2 in cancer. Pharmacol Res. 2008; 58:262-70.

11. Cullinan SB, Diehl JA. Coordination of ER and oxidative stress signaling: the PERK/Nrf2 signaling pathway. Int J Biochem Cell Biol. 2006; 38:317-32.

12. So AY, de la Fuente E, Walter P, Shuman M, Bernales S. The unfolded protein response during prostate cancer development. Cancer Metastasis Rev. 2009; 28: 219-23.

13. Ma Y, Hendershot LM. The role of the unfolded protein response in tumour development: friend or foe? Nat Rev Cancer. 2004; 4: 966-77.

14. Associazione italiana registri tumori (AIRTUM). 2015. http://www.registri-tumori.it/cms/.

15. Strope JD, Price DK, Figg WD. Building a hit list for the fight against metastatic castration resistant prostate cancer. Cancer Biol Ther. 2016; 17:231-2.

16. Hetz C. The unfolded protein response: controlling cell fate decisions under ER stress and beyond. Nat Rev Mol Cell Biol. 2012; 13:89-102.

17. Sheng X, Arnoldussen YJ, Storm M, Tesikova M, Nenseth HZ, Zhao S, Fazli L, Rennie P, Risberg B, Waehre H, Danielsen H, Mills IG, Jin Y, et al. Divergent androgen regulation of unfolded protein response pathways drives prostate cancer. EMBO Mol Med. 2015; 7:788-801.

18. Li J, Lee AS. Stress induction of GRP78/BiP and its role in cancer. Curr Mol Med. 2006; 6:45-54.

19. Ni M, Zhang Y, Lee AS. Beyond the endoplasmic reticulum: atypical GRP78 in cell viability, signalling and therapeutic targeting. Biochem J. 2011; 434: 181-8.

20. de Ridder GG, Ray R, Pizzo SV. A murine monoclonal antibody directed against the carboxyl-terminal domain of GRP78 suppresses melanoma growth in mice. Melanoma Res. 2012; 22:225-35.

21. Miyake H, Hara I, Arakawa S, Kamidono S. Stress protein GRP78 prevents apoptosis induced by calcium ionophore, ionomycin, but not by glycosylation inhibitor, tunicamycin, in human prostate cancer cells. J Cell Biochem. 2000; 77:396-408.
22. Misra UK, Mowery Y, Kaczowka S, Pizzo SV. Ligation of cancer cell surface GRP78 with antibodies directed against its $\mathrm{COOH}$-terminal domain up-regulates p53 activity and promotes apoptosis. Mol Cancer Ther. 2009; 8:1350-62.

23. Wang M, Kaufman RJ. The impact of the endoplasmic reticulum protein-folding environment on cancer development. Nature Rev Cancer. 2014; 14:581-97.

24. Storm M, Sheng X, Arnoldussen YJ, Saatcioglu F. Prostate cancer and the unfolded protein response. Oncotarget. 2016; 7:54051-66. doi: 10.18632/oncotarget.9912.

25. Stachel I, Geismann C, Aden K, Deisinger F, Rosenstiel P, Schreiber S, Sebens S, Arlt A, Schäfer H. Modulation of nuclear factor E2-related factor-2 (Nrf2) activation by the stress response gene immediate early response-3 (IER3) in colonic epithelial cells: a novel mechanism of cellular adaption to inflammatory stress. J Biol Chem. 2014; 289:1917-29.

26. Reddy NM, Potteti HR, Vegiraju S, Chen HJ, Tamatam CM, Reddy SP. PI3K-AKT Signaling via Nrf2 Protects against Hyperoxia-Induced Acute Lung Injury, but Promotes Inflammation Post-Injury Independent of Nrf2 in Mice. PLoS One. 2015; 10:e129676.

27. Shukla S, Maclennan GT, Marengo SR, Resnick MI, Gupta S. Constitutive activation of P I3 K-Akt and NF-kappaB during prostate cancer progression in autochthonous transgenic mouse model. Prostate. 2005; 64:224-39.

28. Misra UK, Deedwania R, Pizzo SV. Activation and crosstalk between Akt, NF-kappaB, and unfolded protein response signaling in 1-LN prostate cancer cells consequent to ligation of cell surface-associated GRP78. J Biol Chem. 2006; 281:13694-707.

29. Misra UK, Pizzo SV. Receptor-recognized $\alpha_{2}$-macroglobulin binds to cell surface-associated GRP78 and activates mTORC1 and mTORC2 signaling in prostate cancer cells. PLoS One. 2012; 7:e51735.

30. Asplin IR, Misra UK, Gawdi G, Gonzalez-Gronow M, Pizzo SV. Selective upregulated expression of the alpha2macroglobulin signaling receptor in highly metastatic 1-LN prostate carcinoma cells. Arch Biochem Biophys. 2000; 383:135-41.

31. Misra UK, Payne S, Pizzo SV. Ligation of prostate cancer cell surface GRP78 activates a proproliferative and antiapoptotic feedback loop: a role for secreted prostatespecific antigen. J Biol Chem. 2011; 286:1248-59.

32. Zhang Y, Liu R, Ni M, Gill P, Lee AS. Cell surface relocalization of the endoplasmic reticulum chaperone and unfolded protein response regulator GRP78/BiP. J Biol Chem. 2010; 285:15065-75.

33. Zhang Y, Tseng CC, Tsai YL, Fu X, Schiff R, Lee AS. Cancer cells resistant to therapy promote cell surface relocalization of GRP78 which complexes with PI3K and enhances PI(3,4,5)P3 production. PloS one. 2013; 8:e80071.

34. Sprangers J, Rabouille C. SEC16 in COPII coat dynamics at ER exit sites. Biochem Soc Trans. 2015; 43:97-103. 
35. Misra UK, Pizzo SV. Activated $\alpha 2$-macroglobulin binding to cell surface GRP78 induces T-loop phosphorylation of Akt1 by PDK1 in association with Raptor. PLoS One. 2014; 9:e88373.

36. Kassi E, Moutsatsou P. Glucocorticoid receptor signaling and prostate cancer. Cancer Lett. 2011; 302:1-10.

37. Hanahan D, Weinberg RA. Hallmarks of cancer: the next generation. Cell. 2011; 144:646-74.

38. Grasso CS, Wu YM, Robinson DR, Cao X, Dhanasekaran SM, Khan AP, Quist MJ, Jing X, Lonigro RJ, Brenner JC, Asangani IA, Ateeq B, Chun SY, et al. The mutational landscape of lethal castration-resistant prostate cancer. Nature. 2012; 487: 239-43.

39. Costanzi E, Urbanelli L, Bellezza I, Magini A, Emiliani C, Minelli A. Hypermethylation contributes to downregulation of lysosomal $\beta$-hexosaminidase $\alpha$ subunit in prostate cancer cells. Biochimie. 2014; 101:75-82.

40. Wyatt AW, Gleave ME. Targeting the adaptive molecular landscape of castration-resistant prostate cancer. EMBO Mol Med. 2015; 7:878-94.

41. Mitsuishi Y, Motohashi H, Yamamoto M. The Keap1-Nrf2 system in cancers: stress response and anabolic metabolism. Front Oncol. 2012; 2:200

42. Bellezza I, Mierla AL, Minelli A. Nrf2 and NF- $\kappa$ B and Their Concerted Modulation in Cancer Pathogenesis and Progression. Cancers (Basel). 2010; 2:483-97.

43. Jaramillo MC, Zhang DD. The emerging role of the Nrf2Keap1 signaling pathway in cancer. Genes Dev. 2013; 27:2179-91.

44. Zhang P, Singh A, Yegnasubramanian S, Esopi D, Kombairaju P, Bodas M, Wu H, Bova SG, Biswal S. Loss of Kelch-like ECH-associated protein 1 function in prostate cancer cells causes chemoresistance and radioresistance and promotes tumor growth. Mol Cancer Ther. 2010; 9:336-46.

45. Hanada N, Takahata T, Zhou Q, Ye X, Sun R, Itoh J, Ishiguro A, Kijima H, Mimura J, Itoh K, Fukuda S, Saijo Y. Methylation of the KEAP1 gene promoter region in human colorectal cancer. BMC Cancer. 2012; 12:66. doi: 10.1186/1471-2407-12-66.

46. MacLeod AK, Acosta-Jimenez L, Coates PJ, McMahon M, Carey FA, Honda T, Henderson CJ, Wolf CR. Aldoketo reductases are biomarkers of NRF2 activity and are co-ordinately overexpressed in non-small cell lung cancer. Br J Cancer. 2016; 115:1530-39.
47. Zheng HC, Takahashi H, Li XH, Hara T, Masuda S, Guan YF, Takano Y. Overexpression of GRP78 and GRP94 are markers for aggressive behavior and poor prognosis in gastric carcinomas. Hum Pat. 2008; 39:1042-49.

48. Auf G, Jabouille A, Guerit S, Pineau R, Delugin M, Bouchecareilh M, Magnin N, Favereaux A, Maitre M, Gaiser T, von Deimling A, Czabanka M, Vajkoczy P, et al. Inositol-requiring enzyme lalpha is a key regulator of angiogenesis and invasion in malignant glioma. Proc Natl Acad Sci U S A. 2010; 107:15553-58.

49. Tang CH, Ranatunga S, Kriss CL, Cubitt CL, Tao J, PinillaIbarz JA, Del Valle JR, Hu CC. Inhibition of ER stressassociated IRE-1/XBP-1 pathway reduces leukemic cell survival. J Clin Invest. 2014; 124:2585-98.

50. Azad AA, Zoubeidi A, Gleave ME, Chi KN. Targeting heat shock proteins in metastatic castration-resistant prostate cancer. Nat Rev Urol. 2015; 12:26-36.

51. Pootrakul L, Datar RH, Shi SR, Cai J, Hawes D, Groshen $\mathrm{SG}$, Lee AS, Cote RJ. Expression of stress response protein Grp78 is associated with the development of castration-resistant prostate cancer. Clin Cancer Res. 2006; 12:5987-93.

52. Fu Y, Wey S, Wang M, Ye R, Liao CP, Roy-Burman P, Lee AS. Pten null prostate tumorigenesis and AKT activation are blocked by targeted knockout of ER chaperone GRP78/ BiP in prostate epithelium. Proc Natl Acad Sci U S A. 2008; 105:19444-49.

53. Culig Z, Santer FR. Androgen receptor signaling in prostate cancer. Cancer Metastasis Rev. 2014; 33:413-27.

54. Bellezza I, Tucci A, Galli F, Grottelli S, Mierla AL, Pilolli $\mathrm{F}$, Minelli A. Inhibition of NF- $\kappa \mathrm{B}$ nuclear translocation via HO-1 activation underlies $\alpha$-tocopheryl succinate toxicity. J Nutr Biochem. 2012; 23:1583-91.

55. Minelli A, Bellezza I, Tucci A, Rambotti MG, Conte C, Culig Z. Differential involvement of reactive oxygen species and nucleoside transporters in cytotoxicity induced by two adenosine analogues in human prostate cancer cells. Prostate. 2009; 69:538-47.

56. Bellezza I, Roberti R, Gatticchi L, Del Sordo R, Rambotti MG, Marchetti MC, Sidoni A, Minelli A. A novel role for $\mathrm{Tm} 7 \mathrm{sf} 2$ gene in regulating TNF $\alpha$ expression. PLoS One. 2013; 8:e68017. 